\title{
Dietary Exposure Risk Assessment of Flonicamid and Its Effect on Constituents after Application in Lonicerae Japonicae Flos
}

\author{
Jiaxin Li, ${ }^{a, \#}$ Yujie Wang, ${ }^{a, \#}$ Jian Xue, ${ }^{* a}$ Pengsi Wang, ${ }^{a}$ and Shangmei Shi*,b \\ ${ }^{a}$ Institute of Medicinal Plant Development, Chinese Academy of Medical Science \& Peking Union Medical College; \\ Beijing 100193, P. R. China: and ${ }^{b}$ Chinese Pharmacopoeia Commission; Beijing 100061, P. R. China. \\ Received December 6, 2017; accepted March 1, 2018; advance publication released online March 14, 2018
}

To investigate the dietary exposure risk of flonicamid application on Lonicerae Japonicae Flos and the effect of flonicamid on constituents of Lonicerae Japonicae Flos, field experiments were conducted in Fengqiu, Henan province, and flonicamid residue in samples collected was detected by gas chromatography equipped with electron capture detector (GC-ECD). And chlorogenic acid and luteoloside were determined by HPLC. Dietary exposure risk assessment was conducted through comparing the estimated daily intake (EDI) which was calculated by using the consumed residual level along with the acceptable daily intake (ADI). The effect of flonicamid on chlorogenic acid and luteoloside were obtained by ANOVA statistical analysis and least significant difference (LSD)- $t$ test. The results showed that the terminal-residue contents of flonicamid were under $1.6 \mathrm{mg} \mathrm{kg}^{-1}$. And risk quotient ranged from 0.0011 to 0.0028 , indicating the long-term exposure to flonicamid residual through consumption of Lonicerae Japonicae Flos in consumers was relatively low. Flonicamid could suppress the generation of luteoloside, so it was not advised to be used in L. japonica flowering phase. The study aims at providing the useful suggestion on the reasonable flonicamid usage and the reference for the establishment of maximum residue limits (MRLs) of flonicamid in Lonicerae Japonicae Flos.

Key words Lonicerae Japonicae Flos; flonicamid; dietary exposure risk assessment; constituent; terminal pesticide residue

Lonicerae Japonicae Flos, also known as Jinyinhua or Japanese honeysuckle, is the dried flower buds or open flowers of Lonicera japonica ThunB. It is one of the most common Traditional Chinese Medicines (TCMs), which has been applied in the healthcare of China and other East Asia countries for many years. And it is proved to have the effect of anticarcinogenic, anti-inflammatory, analgesic, antipyretic, ${ }^{1)}$ antioxidant, ${ }^{2)}$ antiviral, ${ }^{3)}$ and antimicrobial ${ }^{4)}$ functions. Besides, it also has been widely applied in the healthcare along with the cosmetics industries. ${ }^{5,6)}$ Therefore, to meet the growing demand of supply, the cultivation of Lonicerae Japonicae Flos is increasing. But L. japonica is severely damaged by pest and disease every year especially aphis from April to June, that lead to the reduction and quality deterioration of Lonicerae Japonicae Flos. So the use of pesticide is necessary to prevent the damage of aphides.

Flonicamid ( $N$-cyanomethyl-4-trifluoromethylnicotinamide, developed by Ishihara Sangyo Kaisha, Japan) is a novel systemic pesticide which possesses selective toxicity to hemipterous insects, like aphides and whiteflies. Because of the characteristics of fast degradation and low residue, it has a wide usage in Lonicerae Japonicae Flos. Terminal residues or dietary exposure risk assessments of flonicamid in Lonicerae Japonicae Flos have been rarely evaluated. Thus, in order to guarantee the safety use of Lonicerae Japonicae Flos, risk assessment of Lonicerae Japonicae Flos after flonicamid application is necessary.

Chlorogenic acid (CA) and luteoloside are the quality control constituents of Lonicerae Japonicae Flos according to the China Pharmacopoeia ${ }^{7)}$ whose content can reflect the quality of the medical plant. Some studies show that pesticide can

\footnotetext{
\# These authors contributed equally to this work.

affect the constituents of plants. For example, acetamiprid and triadimefon have been proven to have significant effect on chlorogenic acid content. ${ }^{8)}$ And the effect on content of activity components of Lonicerae Japonicae Flos has not been published until now.

So this study aims: (1) to investigate the dietary exposure risk of Lonicerae Japonicae Flos basing on terminal residues after flonicamid application; (2) to study the effect of flonicamid on CA and luteoloside in Lonicerae Japonicae Flos; (3) to provide the advices on rational usage of insecticide and the reference for establishment of maximum residue limits (MRLs) in Lonicerae Japonicae Flos.

\section{Experimental}

Standards, Reagents, Instruments Reference standard of flonicamid (100 mg/L) was purchased from AccuStandard, Inc. (U.S.A.). The standards of CA and luteoloside were obtained from Shanghai Aladdin Bio-Chem Technology Company, Ltd. (China).

Analytical grade solvents, including ethanol, acetonitrile, toluene, glacial acetic acid, and anhydrous sodium sulfate, were obtained from Beijing Chemical Works Company (China). HPLC-grade acetone, acetonitrile were obtained from Fisher Scientific (U.K.). The $\mathrm{NH}_{2}(500 \mathrm{mg}, 6 \mathrm{~mL})$ SPE cartridges $(500 \mathrm{mg}, 6 \mathrm{~mL})$ was got from Anpel Laboratory Technologies Inc. (China).

The gas chromatography (GC) analyses were performed by $6890 \mathrm{~N}$ gas chromatography equipped with $\mu$-electron capture detector (ECD) (Agilent Technologies, U.S.A.). And the column DB-1701 $(30 \mathrm{~m} \times 0.32 \mathrm{~mm} \times 0.25 \mu \mathrm{m}$, Agilent Technologies $)$ was applied for separation. HPLC analyses were performed using the e2695 liquid chromatograph and 2996 potato dextrose agar, or photo diode array (PDA) detector (Waters, 
U.S.A.). Separation was achieved by using an Rsprosil-Pur $120 \mathrm{C}_{18}$ column $(4.6 \times 250 \mathrm{~mm}, 5 \mu \mathrm{m}$, Dr. Maisch, German). Meanwhile, the ultrasonic cleaner (SB-2500DT, Ningbo Scientz Biotechnology Company, China), the vortex mixer (WH-1, Shanghai Huxi Analytical Instruments, China), the rotary vacuum evaporator (LABOROTA-4000, Heidolph Inc., Germany), the vacuum pump (SHB-B, Zhengzhou Greatwall Scientific Company, China) and the centrifuge (80-2, Jintan Medical-Equipment Company, China) were used, too.

Field Trials Field experiments were implemented in Dongzhonggong village, Chengu town, Fengqiu county, Henan province in 2016 springtime, from April 21 to May 23, and were devised on the grounds of 'Guideline on pesticide residue trials' published by Ministry of Agriculture of China. ${ }^{9)}$ In the duration of experiments, the daily minimum temperature was in the range of 11 to $19^{\circ} \mathrm{C}$, and maximum temperature was from 20 to $34^{\circ} \mathrm{C}$. Small rain occurred on May 5, 9, 12 and 23, and moderate rain to small rain occurred on May 14. Moreover, wind strength is no more than 3. Each trial plot included 12 plants of $L$. japonica and the area was about $16.8 \mathrm{~m}^{2}$.

For terminal residue experiment on L. japonica flonicamid ( $10 \%$, Water dispersible granule) was sprayed at two dose levels of $60 \mathrm{~g}$ of active ingredient per hectare ( $\mathrm{g}$ a.i./ha, the recommended dosage) and $90 \mathrm{~g}$ a.i./ha $(1.5 \times$ of the recommended dosage) by application once. It was applied before flowering phase. Each dose level was sprayed 2 times (April 29 and May 6) and 3 times (April 21, 28 and May 5) with 7d interval, respectively. There was a small rain $2 \mathrm{~h}$ later after flonicamid application on May 5. Every treatment had 3 repeated plots. Controls were maintained without pesticide application. Representative flower buds of $L$. japonica were gathered at $5: 30$ of $3 \mathrm{rd}, 7 \mathrm{th}, 12 \mathrm{th}$ and $15 \mathrm{th}$ days after last usage of pesticide. About $100 \mathrm{~g}$ fresh samples were picked randomly in every plot and dried immediately in a drying-oven about $24 \mathrm{~h}$ at $55^{\circ} \mathrm{C}$, then stored in refrigerator at $-20^{\circ} \mathrm{C}$ before analysis.

For the experiment of effect on constituents, the rate of flonicamid was $180 \mathrm{~g}$ a.i./ha ( $3 \times$ of the recommended dosage) by spraying once before flowering phase on May 9. A small rain occurred $3 \mathrm{~h}$ after flonicamid application. The treatment was designed with three replicated plots. Controls were maintained without pesticide application. One hundred grams representative flower buds were picked at $0(2 \mathrm{~h}), 1,2,3,4,6,8$, 10,12 , and $15 \mathrm{~d}$ after pesticide application in every plot. Then all the fresh samples were dried in the same way as above.

\section{Sample Analysis Methods}

\section{Determination of Flonicamid Residues}

Flonicamid in Lonicerae Japonicae Flos was determined according to the methods established in our laboratory. ${ }^{10)}$ One gram of Lonicerae Japonicae Flos powder was taken in $10 \mathrm{~mL}$ centrifuge tube and extracted with $5 \mathrm{~mL}$ acetonitrile, then shaken in a vortex mixer for $5 \mathrm{~min}$ vigorously. The mixture was separated in a centrifuge at $3000 \mathrm{rpm}$ for $15 \mathrm{~min}$ and the supernatant liquid was transferred into a $100 \mathrm{~mL}$ pear-form flask. Five milliliters acetonitrile was used to extract once as above. The supernatants were merged and concentrated to a little milliliter in a vacuum rotatory evaporator at about $40^{\circ} \mathrm{C}$. Next, $5 \mathrm{~mL}$ acetonitrile-toluene $(3: 1, \mathrm{v} / \mathrm{v})$ was added into $\mathrm{NH}_{2} \mathrm{SPE}$ cartridge prior to concentrate addition. Another same flask was connected to cartridge when the added solution attained the top of the sorbent, and the concentrate was injected into cartridge. The container was cleaned three times by using $1 \mathrm{~mL}$ acetonitrile, meanwhile, all washings were put into the cartridge, too. Fifteen milliliters acetonitrile-toluene $(3: 1, \mathrm{v} / \mathrm{v})$ was used to elute the pesticide and the eluate was concentrated to desiccation at $40^{\circ} \mathrm{C}$. Lastly, the residual was redissolved in $2 \mathrm{~mL}$ acetone for $\mathrm{GC}$ determination.

$\mathrm{GC}$ analysis was performed by the usage of temperature programming. The temperature was $120^{\circ} \mathrm{C}$ initially (held $5 \mathrm{~min}$ ), and ramped at a ratio of $15^{\circ} \mathrm{C} / \mathrm{min}$ to $190^{\circ} \mathrm{C}$ (held $1 \mathrm{~min}$ ), then ramped at $20^{\circ} \mathrm{C} / \mathrm{min}$ to the last $260^{\circ} \mathrm{C}$ (held $1 \mathrm{~min}$ ). Nitrogen (high purity, 99.999\%) was applied as carrier gas and the flow was $1 \mathrm{~mL} / \mathrm{min}$. The injector temperature was $260^{\circ} \mathrm{C}$ in splitless mode, and injection volume was $1 \mu \mathrm{L}$. The detector was set at a temperature of $320^{\circ} \mathrm{C}$.

Determination of CA and Luteoloside

The CA and luteoloside in Lonicerae Japonicae Flos were determined according to the method in Chinese Pharmacopeia (ChP). One gram of sample powder was homogenized in $20 \mathrm{~mL} 70 \%(\mathrm{v} / \mathrm{v})$ ethanol, and ultrasonic extraction was carried out for $60 \mathrm{~min}$. A $0.45 \mu \mathrm{m}$ membrane filter was used to filtrate the supernatant before injecting. Assay was performed in a HPLC system. The injection volume was $10 \mu \mathrm{L}$. The mobile phase was constituted by $0.5 \%(\mathrm{v} / \mathrm{v})$ glacial acetic acid aqueous solution (A) and acetonitrile (B), and a gradient elution procedure was applied as follows: at $0 \mathrm{~min}$, the volume ratio between $\mathrm{A}$ and $\mathrm{B}$ was $95 / 5$, and the ratio was changed to $87 / 13$ from 0 to $7 \mathrm{~min}$, subsequently to $82 / 18$ from 7 to $43 \mathrm{~min}$, and to $70 / 30$ from 43 to $55 \mathrm{~min}$, to $5 / 95$ from 55 to $60 \mathrm{~min}$, finally returned to $95 / 5$ at $65 \mathrm{~min}$ through a linear gradient. The velocity of flow was $1 \mathrm{~mL} / \mathrm{min}$, and the temperature of column was $25^{\circ} \mathrm{C}$. CA and luteoloside were detected at $350 \mathrm{~nm}$. And the standards containing $50.0 \mu \mathrm{g}$ CA and $42.0 \mu \mathrm{g}$ Luteoloside per milliliter was used for the analysis.

\section{Statistical Analysis}

\section{Dietary Exposure Risk Assessment}

The calculations of dietary exposure and risk assessment were utilized the equations as follows.

$$
\mathrm{EDI}=\frac{F_{i} \times R L_{i}}{\text { mean body weight }}
$$

EDI, $F_{i}$ and $R L_{i}$ represented estimated daily intake $(\mathrm{mg} / \mathrm{kg}$ body weight (b.w.)), food consumption data (g/d) and residue level for commodity $(\mathrm{mg} / \mathrm{kg})$, respectively. If the results were lower than the limit of quantitation (LOQ) of analytical method, the calculations of intake were employed LOQ values. ${ }^{11)}$

$$
R Q=\frac{\mathrm{EDI}}{\mathrm{ADI}}
$$

$R Q$ was the value of risk quotient, and ADI was acceptable daily intake (mg/kg b.w.). If the value of $R Q$ was more than 1 , it manifested that the risk of pesticide was unacceptable in human. By comparison, if the value was less than 1, it indicated minimal risk to human. ${ }^{12)}$

Effect of Flonicamid on CA and Luteoloside

The ANOVA statistical analysis and least significant difference (LSD)- $t$ test were carried out to find differences between the treatments by IBM SPSS Statistics 22.

\section{Results and Discussion \\ Terminal Residues of Flonicamid in Lonicerae Japoni- cae Flos The terminal residuals of flonicamid in Lonicerae}




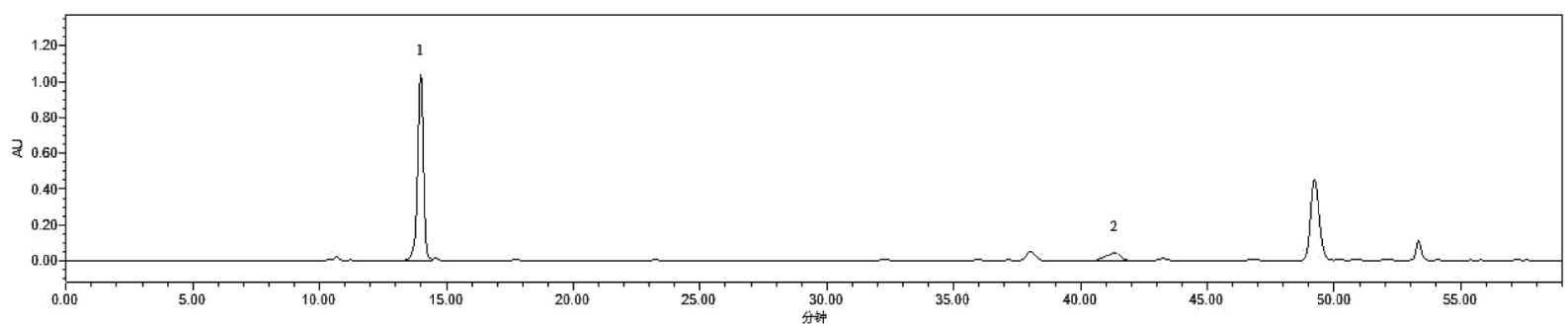

Fig. 1. HPLC Chromatograms of Lonicerae Japonicae Flos Sample

1: Chlorogenic acid. 2: Luteoloside.

Table 1. Terminal Residues of Flonicamid in Lonicerae Japonicae Flos

\begin{tabular}{cccc}
\hline $\begin{array}{c}\text { Application dose } \\
\text { (g a.i./ha) }\end{array}$ & $\begin{array}{c}\text { Number of } \\
\text { applications }\end{array}$ & PHI (d) & Residues (mg/kg) \\
\hline 60 & 2 & 3 & $0.71-0.83$ \\
& 7 & $0.30-0.54$ \\
& 12 & $0.091-0.11$ \\
& 17 & $0.019-0.029$ \\
& 3 & 7 & $0.60-0.76$ \\
& & 12 & $0.20-0.31$ \\
& & 17 & $0.080-0.88$ \\
& 30 & 7 & $0.017-0.028$ \\
& & $1.5-1.6$ \\
& & 17 & $0.63-0.72$ \\
& & 3 & $0.088-0.3$ \\
& 7 & $0.040-0.043$ \\
& 12 & $0.69-0.97$ \\
& 17 & $0.15-0.43$ \\
& & $0.075-0.092$ \\
& & $0.020-0.028$ \\
\hline
\end{tabular}

Table 2. Risk of Flonicamid in Lonicerae Japonicae Flos

\begin{tabular}{cccccc}
\hline \hline $\begin{array}{c}\text { b.w. } \\
(\mathrm{kg})\end{array}$ & $\begin{array}{c}F_{i} \\
(\mathrm{~g} / \mathrm{d})\end{array}$ & $\begin{array}{c}R L \\
(\mathrm{mg} / \mathrm{kg})\end{array}$ & $\begin{array}{c}\text { EDI } \\
(\mu \mathrm{g} / \mathrm{kg} \text { b.w. })\end{array}$ & $\begin{array}{c}\text { ADI } \\
(\mathrm{mg} / \mathrm{kg} \text { b.w. })\end{array}$ & $R Q$ \\
\hline 60 & $6-15$ & 0.78 & $0.078-0.195$ & 0.07 & $0.0011-0.0028$ \\
\hline
\end{tabular}

Table 3. Contents of Chlorogenic Acid and Luteoloside in Control Plots and Flonicamid Treat Plots $(n=3)$, and ANOVA Results

\begin{tabular}{lccccc}
\hline \hline \multirow{2}{*}{$\begin{array}{c}\text { Days after } \\
\text { flonicamid spray }(\mathrm{d})\end{array}$} & \multicolumn{2}{c}{ Chlorogenic acid (\%) } & & \multicolumn{2}{c}{ Luteoloside (\%) } \\
\cline { 6 - 7 } \cline { 5 - 6 } & $\left.\mathrm{T}^{a}{ }^{a}\right)$ & $\mathrm{T} 2^{b)}$ & & $\mathrm{T} 1$ & $\mathrm{~T} 2$ \\
\hline 2 & $2.45 \pm 0.02$ & $2.50 \pm 0.03$ & & $0.094 \pm 0.003$ & $0.079 \pm 0.004$ \\
4 & $2.38 \pm 0.01$ & $2.43 \pm 0.04$ & & $0.083 \pm 0.002$ & $0.071 \pm 0.002$ \\
6 & $2.56 \pm 0.03$ & $2.55 \pm 0.03$ & & $0.096 \pm 0.002$ & $0.061 \pm 0.001$ \\
8 & $2.55 \pm 0.03$ & $2.61 \pm 0.03$ & & $0.089 \pm 0.003$ & $0.060 \pm 0.003$ \\
10 & $2.54 \pm 0.03$ & $2.78 \pm 0.04$ & & $0.089 \pm 0.002$ & $0.088 \pm 0.002$ \\
12 & $2.49 \pm 0.01$ & $2.59 \pm 0.02$ & & $0.085 \pm 0.002$ & $0.076 \pm 0.002$ \\
15 & $2.57 \pm 0.02$ & $2.45 \pm 0.03$ & & $0.089 \pm 0.002$ & $0.083 \pm 0.001$ \\
Average & 2.51 & 2.56 & & 0.089 & 0.074 \\
$p$ Value & \multicolumn{3}{c}{0.330} & & \multicolumn{2}{c}{$0.004^{c)}$} \\
Result & No significant influence & Decrease significantly \\
\hline
\end{tabular}

a) T1: Control plots. b) T2: Flonicamid treat plots. c) Difference is significant at the 0.01 level.

Japonicae Flos were determined according to the methods above, and the gas chromatogram of the solution of flonicamid standard and honeysuckle sample could refer to Fig. 2 in our article. ${ }^{10)}$ Table 1 summarized the results of terminal residues.

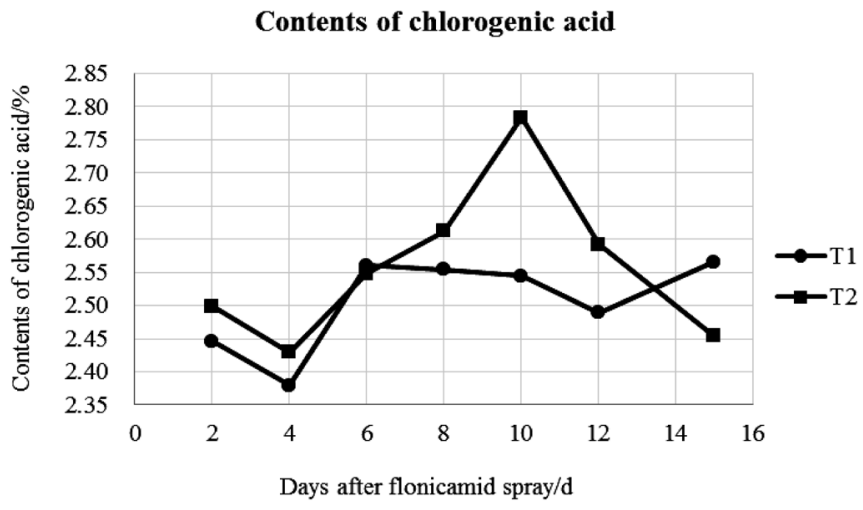

Fig. 2. Contents of Chlorogenic Acid

T1: Control plots. T2: Flonicamid treated plots.

\section{Contents of luteoloside}

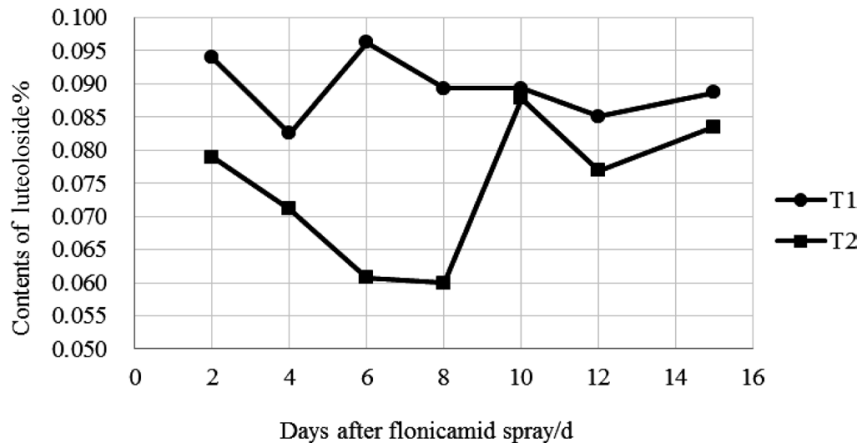

Fig. 3. Contents of Luteoloside

T1: Control plots. T2: Flonicamid treated plots.

The results for the number of applications 3 might be impacted by a small rain occurred $2 \mathrm{~h}$ after flonicamid application on May 5. The small rain might wash away some of the unabsorbed flonicamid on the surface of plant. Moreover, as too many applications, the dose level of 60 was sprayed firstly at 6:00 and dose 90 was not sprayed until 11:00. It might have a greater washing effect on the plots of dose 90 since the time of flonicamid application was closer to that of rain. Thus, the effect on the detected results for dose 90 might also be greater.

On the basis of these data, the terminal residues of flonicamid were no more than $1.6 \mathrm{mg} / \mathrm{kg}$. All these residues were under MRL calculated according to the $\mathrm{ChP}(2.8 \mathrm{mg} / \mathrm{kg})$. The result indicated that the residual level rested with application dose and pre-harvest interval (PHI) value. The residual level decreased along with the fewer dose of application, for an- 
other, it also reduced with the increasing PHI when the same dose and number of applications were used. The conclusion was same as that Shi et al. ${ }^{13)}$ got from eggplants.

Dietary Exposure Risk Assessment Long-term dietary exposure risk demanded to contrast the value of EDI and ADI. Average b.w. was $60 \mathrm{~kg}$ and Lonicerae Japonicae Flos intake was $0.006-0.015 \mathrm{~kg}$ according to $\mathrm{ChP}$. The ADI value for flonicamid was $0.07 \mathrm{mg} / \mathrm{kg}$ b.w. ${ }^{14}$

The result of terminal residues showed that the residual dynamics of flonicamid following a tendency of shorter PHI resulted in greater residues. Therefore, the risk should be low if the assessment result related to the shortest PHI $(3 \mathrm{~d})$ was acceptable. At 3rd day, the flonicamid residuals for each treatment of 3 replications were $0.60,0.69,0.71,0.72,0.74,0.76$, $0.80,0.83,0.97,1.5,1.6,1.6 \mathrm{mg} / \mathrm{kg}$, respectively. In the light of the Supervised Trial Median Residue (STMR), the $R L$ of flonicamid was $0.78 \mathrm{mg} / \mathrm{kg}$. Table 2 summarized the dietary exposure risk assessment of flonicamid in Lonicerae Japonicae Flos. As table shown, the EDI of flonicamid ranged from $0.078-0.195 \mu \mathrm{g} / \mathrm{kg}$ b.w.

The $R Q$ ranged from 0.0011 to 0.0028 which was far lower than 1 . The result indicated that the long-term exposure of flonicamid residues to Lonicerae Japonicae Flos consumers was relatively low under the recommended dosage and PHI. So it suggested that using flonicamid not more than $90 \mathrm{~g}$ a.i./ha for less than 3 times cannot lead to dietary risk.

Contents of CA and Luteoloside in Lonicerae Japonicae Flos The samples treated by flonicamid and the control were determined by the method as above. Figure 1 showed the HPLC chromatograms of Lonicerae Japonicae Flos sample. Table 3, Figs. 2 and 3 presented the content of CA and luteoloside in samples of control plots and flonicamid treat plots. The data of samples picked in $0(2 \mathrm{~h}), 1$ and $3 \mathrm{~d}$ were removed, because these samples were damaged during drying, they could not be used as representative samples in content analysis of constituents. As the table and figures showed, the content of CA ranged from 2.38 to $2.57 \%$ in control plots and from 2.43 to $2.78 \%$ in flonicamid disposed plots. While, the content of luteoloside ranged from 0.083 to $0.094 \%$ in control plots and 0.061 to $0.088 \%$ in flonicamid disposed plots. Since the flowering phase of L. japonica was long, for this medicinal material, it was appropriate to evaluate the average quality of samples harvested during the flowering phase rather than the quality of samples picked on a certain day. The content of $\mathrm{CA}$ and luteoloside met the requirements of ChP. And it was clear that the content of CA increased and that of luteoloside decreased.

The Results of ANOVA Statistical Analysis between the Treatments The results of ANOVA statistical analysis by IBM SPSS Statistics 22 was shown in Table 3. As table shown, the CA content in Lonicerae Japonicae Flos applied flonicamid had no significant differences $(p>0.05)$ compared with control. And there was significant difference on the content of luteoloside $(p<0.01)$ between control and treatment group. The content of luteoloside decreased significantly after pesticide application. So results indicated that flonicamid could suppress the generation of luteoloside, which could affect the quality of Lonicerae Japonicae Flos. So the use of flonicamid was not suitable in the flowering phase of $L$. japonica.

\section{Conclusion}

Terminal residues of flonicamid and dietary exposure risk assessment in Lonicerae Japonicae Flos were investigated. The terminal residue contents of flonicamid were under $1.6 \mathrm{mg} / \mathrm{kg}$. The dietary exposure risk assessment based on $R Q$ value ranged from $0.0011-0.0028$, indicating that the risk of Lonicerae Japonicae Flos after standard flonicamid treatment was low. In addition, the effects of flonicamid on the constituents in Lonicerae Japonicae Flos were studied. Flonicamid could significantly decrease the content of luteoloside, while it had no effect on CA. This conclusion indicated that flonicamid could affect the quality of Lonicerae Japonicae Flos. Thus, the advice that flonicamid cannot be used in flowering phase of $L$. japonica were given. These researches provided some suggestion on the rational usage of flonicamid in L. japonica. What is more, the current study could use for a reference for the MRLs establishment of flonicamid in Lonicerae Japonicae Flos.

Acknowledgment This paper was supported by China Food and Drug Administration, and the Item Number was ZG2016-2.

Conflict of Interest The authors declare no conflict of interest.

\section{References}

1) dos Santos M. D., Almeida M. C., Lopes N. P., De Souza G. E. P., Biol. Pharm. Bull., 29, 2236-2240 (2006).

2) Xu J. G., Hu Q. P., Liu Y., J. Agric. Food Chem., 60, 11625-11630 (2012).

3) Wang G. F., Shi L. P., Ren Y. D., Liu Q. F., Liu H. F., Zhang R. J., Li Z., Zhu F. H., He P. L., Tang W., Tao P. Z., Li C., Zhao W. M., Zuo J. P., Antiviral Res., 83, 186-190 (2009).

4) Shang X., Pan H., Li M., Miao X., Ding H., J. Ethnopharmacol., 138, 1-21 (2011).

5) Zhang B., Yang R., Zhao Y., Liu C. Z., J. Chromatogr. B Analyt. Technol. Biomed. Life Sci., 867, 253-258 (2008).

6) Sun P. C., Liu Y., Yi Y. T., Li H. J., Fan P., Xia C. H., Food Chem., 168, 55-62 (2015).

7) China Pharmacopoeia Committee, "LONICERAE JAPONICAE FLOS. China Pharmacopoeia Part 1,” 2015 Edition, China Medical Science Press, Beijing, China, 2015, p. 221.

8) Wu X., Xue J., Zhang L., Sun N., Liu D., Asian J. Chem., 24, 3829 (2012).

9) Liu G. X., Qiao X. W., Tao C. J., He Y. B., Gong Y., Qin D. M., Zhu G. Y., Qin S., Li Y. S., Song W. C., "Guideline for Pesticide Residue Trials, NY/T 788-2004," The Ministry of Agriculture of the People's Republic of China, Beijing, China, 2003, pp. 1-6.

10) Wang Y. J., Xue J., Jin H. Y., Ma S. C., Chem. Pharm. Bull., 65, 492-497 (2017)

11) Li W., Qiu S. P., Wu Y. J., Ecotoxicol. Environ. Saf., 69, 312-316 (2008).

12) Institute of Quality Standard and Testing Technology for Agroproducts of CAAS, "Risk Assessment for Quality and Safety of Agro-foods: Principles Methodologies and Applications." Standards Press of China, Beijing, China, 2007.

13) Shi K., Li L., Yuan L., Li W., Liu F., Int. J. Environ. Anal. Chem., 96, 173-184 (2016).

14) World Health Organization and Food and Agriculture Organization of the United Nations, "Pesticide residues in food 2015," WHO Press, Geneva, Switzerland, 2015, p. 159. 